\title{
Remembering Luigi Padeletti
}

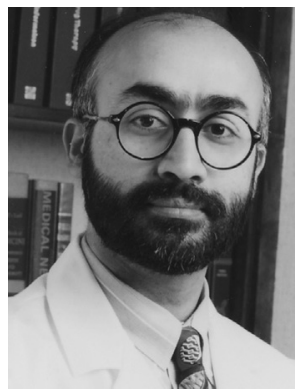

Ranjan K. Thakur, MD, MPH, MBA, FHRS

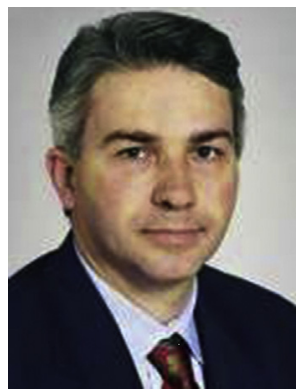

Andrea Natale, MD, FACC, FHRS

Consulting Editors

We introduce this issue of Cardiac Electrophysiology Clinics by expressing our sorrow and paying tribute to an editor, Dr Luigi Padeletti. Dr Padeletti was in the process of editing the current issue when he passed away unexpectedly. We are grateful to his coeditors, Drs Bagliani and De Ponti, who completed what Dr Padeletti could not. Dr Padeletti had been an ardent supporter of the Cardiac Electrophysiology Clinics, having contributed several articles and having edited multiple issues over the years.

Dr Padeletti inspired and edited two recent issues of Cardiac Electrophysiology Clinics; the first issue focused on basic electrocardiography in normal and diseased hearts. That issue took a unique approach to discussing electrocardiography of arrhythmias. It first detailed what can be learned about physiology, pathology, and neural control from each wave and interval of the electrocardiogram and then built on that to discuss arrhythmias originating in each cardiac structure. This companion issue of the Cardiac Electrophysiology Clinics focuses on electrocardiography of various arrhythmias: bradycardias, tachycardias, and some specific arrhythmias.

We and the coeditors of this issue are grateful to have known and worked with $\mathrm{Dr}$ Padeletti and draw inspiration from his example of dedication to scholarship. We hope the readers will enjoy reading and learning from this issue, because that will be the most fitting tribute to his memory.

Ranjan K. Thakur, MD, MPH, MBA, FHRS Sparrow Thoracic and Cardiovascular Institute Michigan State University 1440 East Michigan Avenue, Suite 400 Lansing, MI 48912, USA

Andrea Natale, MD, FACC, FHRS Texas Cardiac Arrhythmia Institute

Center for Atrial Fibrillation at

St. David's Medical Center 1015 East 32nd Street, Suite 516 Austin, TX 78705, USA

E-mail addresses: thakur@msu.edu (R.K. Thakur) andrea.natale@stdavids.com (A. Natale) 